\title{
Passive range of motion exercise to enhance growth in infants following the Norwood procedure: a safety and feasibility trial
}

\author{
Linda M. Lambert, ${ }^{1}$ Felicia L. Trachtenberg, ${ }^{2}$ Victoria L. Pemberton, ${ }^{3}$ Janine Wood, ${ }^{1}$ Shelley Andreas, ${ }^{4}$ \\ Robin Schlosser, ${ }^{4}$ Teresa Barnard, ${ }^{5}$ Kaitlyn Daniels, ${ }^{6}$ Ann T. Harrington, ${ }^{6}$ Nicholas Dagincourt, ${ }^{2}$ \\ Thomas A. Miller ${ }^{7}$ for the Pediatric Heart Network Investigators \\ ${ }^{1}$ Primary Children's Hospital, Salt Lake City, Utab; ${ }^{2}$ New England Research Institutes, Watertown, Massachusetts; \\ ${ }^{3}$ National Heart, Lung, and Blood Institute, Bethesda, Maryland; ${ }^{4}$ Texas Children's Hospital, Houston, Texas; \\ ${ }^{5}$ Cincinnati Children's Hospital Medical Center, Cincinnati, Obio; ${ }^{6}$ The Children's Hospital of Philadelphia, \\ Philadelphia, Pennsylvania; ${ }^{7}$ University of Utah, Salt Lake City, Utah, United States of America
}

\begin{abstract}
Objective: The aim of this study was to evaluate the safety and feasibility of a passive range of motion exercise programme for infants with CHD. Study design: This non-randomised pilot study enrolled 20 neonates following Stage I palliation for single-ventricle physiology. Trained physical therapists administered standardised 15-20-minute passive range of motion protocol, for up to 21 days or until hospital discharge. Safety assessments included vital signs measured before, during, and after the exercise as well as adverse events recorded through the pre-Stage II follow-up. Feasibility was determined by the percent of days that $>75 \%$ of the passive range of motion protocol was completed. Results: A total of 20 infants were enrolled (70\% males) for the present study. The median age at enrolment was 8 days (with a range from 5 to 23), with a median start of intervention at postoperative day 4 (with a range from 2 to 12). The median hospital length of stay following surgery was 15 days (with a range from 9 to 131), with an average of 13.4 (with a range from 3 to 21) in-hospital days per patient. Completion of $>75 \%$ of the protocol was achieved on $88 \%$ of eligible days. Of 11 adverse events reported in six patients, 10 were expected with one determined to be possibly related to the study intervention. There were no clinically significant changes in vital signs. At pre-Stage II follow-up, weight-for-age z-score $(-0.84 \pm 1.20)$ and length-for-age z-score $(-0.83 \pm 1.31)$ were higher compared with historical controls from two earlier trials. Conclusion: A passive range of motion exercise programme is safe and feasible in infants with single-ventricle physiology. Larger studies are needed to determine the optimal duration of passive range of motion and its effect on somatic growth.
\end{abstract}

Keywords: Hypoplastic left heart syndrome; paediatrics; growth; passive range of motion; exercise therapy

Received: 7 November 2016; Accepted: 18 February 2017; First published online: 23 March 2017

G ROWTH IMPAIRMENT IS COMMON IN INFANTS with CHD, especially those with singleventricle physiology. These infants usually undergo three palliative surgeries, with the first Stage I palliation, also called the Norwood surgery, typically within the 1st week of life. Haemodynamic

Correspondence to: L. M. Lambert, MSN-FNP, Division Cardiothoracic Surgery, Primary Children's Hospital, 100 North Mario Capecchi Dr., Salt Lake City, UT 84113, United States of America. Tel: +1 801662 5573; Fax: +1 801662 5571; E-mail: linda.lambert@hsc.utah.edu compromise, inadequate nutrition, and hypoxaemia following surgical repair place a heavy burden on the neonate with limited metabolic reserves. ${ }^{1}$ From birth through 3 years of life, growth rates in children with CHD fall below the norm. ${ }^{2,3}$ Growth failure is manifested by impairment in both length and weight, but initially weight is affected to a larger degree than length. Growth of children with singleventricle physiology has the most dramatic decline with respect to weight-for-age z-scores occurring between birth and discharge after the Norwood 
procedure, up to about 3 months of age, with a relative plateau during the interstage period - that is, the time between Stage I and Stage II palliations., The second palliative surgery - Stage II palliation, also called the Glenn or superior cavopulmonary anastomosis - performed at 3-6 months of age reduces volume load on the ventricle and leads to improved growth trajectories; ${ }^{5}$ however, this can result in catch-up growth that predisposes the child to an increased risk of obesity, diabetes mellitus, hypertension, cardiovascular disease, and osteopaenia later in life. ${ }^{6}$

Comparing infants with a variety of $\mathrm{CHD}$, growth failure is most severe in infants with congestive heart failure and cyanosis. ${ }^{7}$ In infants with hypoplastic left heart syndrome, this appears to be related in part to higher energy expenditure. ${ }^{8}$ Nutritional management alone does not ensure adequate weight gain following the Norwood operation. ${ }^{3,9}$ This is a critically important problem as poor growth and nutrition in early infancy may adversely affect outcomes during subsequent surgical palliation, ${ }^{3,10}$ linear growth, ${ }^{11}$ and impact longterm neurodevelopment. ${ }^{12,13}$

Growth challenges in infants born with single ventricles are similar to those of preterm infants who also experience feeding difficulties, failure to thrive, and subsequent neurodevelopmental impairment. Assisted exercise, passive range of motion therapy, in preterm infants has been shown to increase bone mineral acquisition and bone strength and enhance weight gain. ${ }^{14-16}$ Exercise therapy in preterm infants is also associated with increased somatic growth, ${ }^{17,18}$ improvements in the distribution of sleep-awake states, and decreased behavioural signs of stress. ${ }^{19-21}$ Data regarding the effects of exercise on growth and development are not available for infants with CHD. In fact, most preterm studies have excluded infants with CHD from participation; furthermore, the safety and feasibility of passive range of motion and increased handling of these high-risk infants, often medically unstable, are unknown.

Interventions to increase growth in the singleventricle population have focussed on aggressive nutritional strategies and/or drug therapy with varying degrees of success; however, it is not until Stage II palliation that catch-up growth actually starts to occur. ${ }^{3-5}$ Enhanced growth in the neonatal period may be crucial to favourably impact neurodevelopment, behavioural state, and time to hospital discharge. Changing how caregivers interact with these neonates and tailoring interactions to improve neonatal response may have a positive impact on their development. ${ }^{22}$ Passive range of motion therapy is non-invasive and easy to deliver without adverse effects in premature infants. We hypothesised that a passive range of motion exercise intervention would be safe and feasible in neonates with single-ventricle physiology in the immediate postoperative period after Stage I palliation.

\section{Methods}

\section{Patients}

The "Training in Exercise Activities and Motion for Growth" - TEAM for Growth - study protocol developed by the nursing research committee of the Pediatric Heart Network was approved by the Institutional Review Boards at each of the three Pediatric Heart Network participating centres. The Pediatric Heart Network is a collaboration of clinical sites and a data coordinating centre that conducts research studies on children with CHD or acquired heart disease. Patients were screened for eligibility before approaching the parents for consent (Fig 1). Inclusion criteria included patients $>37$ weeks gestation and $<30$ days of age with hypoplastic left heart syndrome, who had undergone a Norwood procedure for Stage I palliation.

Patients were considered eligible for enrolment once they were haemodynamically stable, as assessed by the attending physician, after surgical intervention with a closed sternum, irrespective of remaining intubated, use of inotropes, and chest tubes and intracardiac lines being in place; feeding status did not play a role in decision making. Exclusion criteria included additional surgical intervention before the Norwood procedure, intrauterine growth restriction, a chromosomal or recognisable phenotypic syndrome associated with growth failure - for example, Trisomy 21, Noonan, or Turner syndromes - a non-cardiac diagnosis associated with growth failure, severe neurological injury resulting from stroke or intracranial haemorrhage confirmed by head CT/MRI or cranial ultrasound, and anticipated discharge within 14 days of screening.

\section{Study design and measures}

This study was a non-randomised, pilot study of an exercise programme in patients with single-ventricle physiology. The primary aim was to determine whether an exercise programme of passive range of motion was safe and feasible in infants with singleventricle physiology following Norwood surgery.

Data collection and time points. Data collected at the time of enrolment included age, race, ethnicity, sex, birth history, and the shunt type used during the Stage 1 operation. Anthropometric measurements such as length and weight were performed by trained nurses using a standardised measurement protocol ${ }^{23}$ at four time points: baseline, 14 days after enrolment, 21 days after enrolment, and at the pre-Stage II 


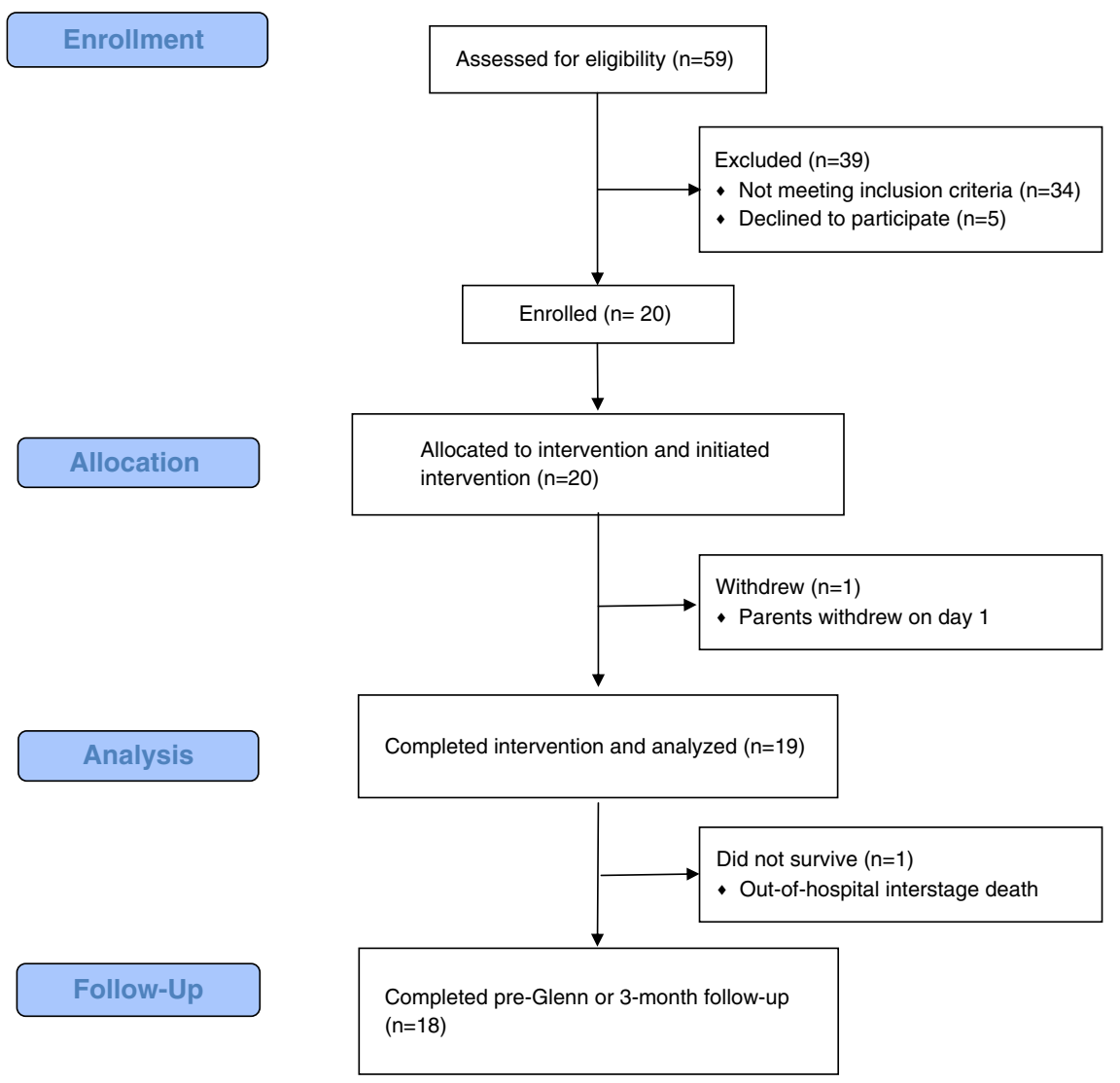

Figure 1.

CONSORT flow diagram: passive range of motion in infants following the Norwood procedure: a safety and feasibility trial.

palliation visit. Day-21 length and weight were collected at the time of hospital discharge if discharge occurred earlier than 21 days. Patients were considered to have completed the study following their anthropometric measurements collected at the pre-Stage II palliation visit.

Passive range of motion exercise. An exercise protocol and training video were developed to assist physical therapists in training. At each site, a lead physical therapist was designated and responsible for training a small group of participating therapists. This physical activity programme was based on the protocol developed by Moyer-Mileur et $\mathrm{al}^{16}$ and adapted by McQueen et $\mathrm{al}^{24}$ as a caregiver-assisted exercise programme for preterm infants. The intervention consisted of a total of 80 repetitions on upper and lower extremities, which included systematic flexion and extension with gentle compression at the end of the movement performed 10 times at each wrist, elbow, shoulder, ankle, knee, and hip joint with adduction and abduction performed 10 times at the shoulder and hip joints. The assisted exercise was performed by a physical therapist 7 seven days a week for 21 consecutive days or until hospital discharge. This once daily, 15-20-minute intervention over 21 days was based on multiple factors. First, there is evidence that a once-a-day regimen is effective in preterm infants. ${ }^{16,17,24}$ Second, the median length of postoperative stay at two of the centres conducting this pilot was at least 24 days. Third, in a fragile singleventricle population, a brief intervention was anticipated to minimise adverse events. Finally, to encourage participation and successfully conduct this study in busy cardiac ICU, any intervention must not be seen as intrusive.

Safety. Infants were monitored before, during, and after the intervention. The infant's heart rate, blood pressure, and oxygen saturation were recorded as a measure of tolerance to the study intervention.

Adverse events were collected up to the final study visit. The number of adverse events was considered a highly clinically relevant measure of safety for this study; however, given the myriad of complications expected to occur during the patients' normal medical course, those that were possibly or probably related to the intervention or those noted to occur during the intervention or for 1 hour after intervention were evaluated to determine the safety of this intervention. At the time, an event was initially reported, and determination of causality was made by the 
designated site principal investigator in consultation with the physical therapist and/or nurse coordinator. The Pediatric Heart Network Medical Monitor independently adjudicated all adverse events for this study and made the final determination of causality and expectedness, which was used in the analysis.

Feasibility. Adherence to the study regimen was assessed daily. The physical therapist recorded the time at which the intervention began and ended, as well as the number of repetitions performed on each extremity. Feasibility was measured by the ability to complete the exercise protocol, and was characterised each day as follows: high completion, defined as $\geqslant 75 \%$ of the intervention performed; medium completion, defined as $50-75 \%$ of the intervention performed; and low completion, defined as $<50 \%$ of the intervention performed.

Anthropometry and growth. Preliminary efficacy of the intervention was considered by assessing anthropometry throughout the study period. Weight-for-age and length-for-age z-scores were calculated using the World Health Organization child growth standards algorithm. Differences in mean weight-for-age and length-for-age z-scores at the pre-Stage II palliation visit were compared with previous Pediatric Heart Network cohorts enrolled in the Infant Single Ventricle Trial and Single Ventricle Reconstruction Trial. ${ }^{25,26}$ Changes in weight-for-age and length-for-age z-scores from baseline to day 21 or discharge, baseline to pre-Stage II, and day 21 or discharge to pre-Stage II were also compared between the three cohorts.

\section{Statistical analysis}

Logistic regression was used to test potential predictors of high/medium versus low completion rate. Changes in vital signs from pre-intervention to during-intervention and from pre-intervention to post-intervention periods were calculated, and a 1 -sample t-test was used to determine statistically significant differences from 0 ; two-sample t-tests were used to compare anthropometry and change in anthropometry between studies. All analyses were conducted using SAS v9.3 (SAS Institute Inc., Cary, North Carolina, United States of America), and statistical significance was tested at the 0.05 level. Owing to the challenges in interpreting the significance of multiple testing, we focus on comparing the number of significant results with that expected because of chance alone. We made 10 vital sign comparisons, eight z-score comparisons, and considered six predictors of completion rate. In these 24 statistical tests, we expect 1-2 significant differences due to chance alone.

\section{Results}

Neonates were enrolled at three Pediatric Heart Network institutions between September, 2013 and September, 2014; 20/25 eligible patients consented $(80 \%)$ to participate and $70 \%$ of them were males (Table 1). After 3 days of intervention, one patient was voluntarily withdrawn by the parents. Another patient died before the 3 -month visit after completing 13 days of the intervention and being discharged home. The remaining patients were followed-up through the pre-Stage II palliation visit. Table 2 describes enrolment and duration of the intervention. Patients were enrolled at a median age of 8 days (with a range from 5 to 23), with the first passive range of motion starting on a median postoperative day 4 (with a range from 2 to 12). The median hospital length of stay following surgery was 15 days (with a range from 9 to 131), allowing for an average of 13.4 in-hospital days/patient (with a range from 3 to 21) for attempted passive range of motion exercise.

Safety

Of 11 adverse events reported in six patients, all were adjudicated to be "expected" by the Pediatric Heart Network Medical Monitor. Only one adverse event, a midsternal wound dehiscence, was determined by the medical monitor to be possibly but not probably related to the study intervention. The top of the incision was noted to be open and draining, with no erythema. The principal investigator related the wound change to a probable suture granuloma or fat necrosis. This patient completed all 21 days of the exercise protocol and had total resolution of the wound complication.

Vital signs were monitored before, during, and after intervention. Although heart rate changes from

Table 1. Demographics of the Training in Exercise Activities and Motion for Growth cohort $(n=20)$.

\begin{tabular}{lc} 
Characteristics & $\mathrm{n}(\%)$ or Median [range] \\
\hline Male (n (\%)) & $14(70 \%)$ \\
Race (n (\%)) & $12(60 \%)$ \\
$\quad$ Caucasian & $1(5 \%)$ \\
$\quad$ African-American & $2(10 \%)$ \\
$\quad$ Asian & $1(5 \%)$ \\
$\quad$ American Indian or Alaska Native & $4(20 \%)$ \\
$\quad$ Unknown & $2(10 \%)$ \\
Ethnicity (n (\%)) & $3.31[2.3-4.59]$ \\
$\quad$ Hispanic or Latino & $50[44.0-57.0]$ \\
Birth weight $(\mathrm{kg})$ & $14(74 \%)$ \\
Birth length $(\mathrm{cm})$ & \\
Right ventricle-to-pulmonary artery & \\
conduit $(\mathrm{n}(\%))$ &
\end{tabular}


Table 2. Timing and duration of the study intervention $(n=20)$.

\begin{tabular}{lc}
\hline Characteristics & Median [range] \\
\hline Age at enrolment (days) & $8[5-23]$ \\
Postoperative day on which the passive range of motion exercise was started & $4[2-12]$ \\
No. of days after enrolment range of motion exercise started & $1[0-3.0]$ \\
Hospital length of stay (days) & $15[9-131]$ \\
Average range of motion (days/patient) & $13.4[3-21]$ \\
Ventilated days receiving range of motion & $1[0-21]$ \\
\hline
\end{tabular}

Table 3. Change in vital signs.

\begin{tabular}{lllll}
\hline & \multicolumn{2}{l}{$\begin{array}{l}\text { Change from before intervention to during } \\
\text { intervention }\end{array}$} & \multicolumn{2}{l}{$\begin{array}{l}\text { Change from before intervention to after } \\
\text { intervention }\end{array}$} \\
\cline { 2 - 3 } Variables & Mean (SD) & p-value* & Mean (SD) & p-value* \\
\hline Heart rate (beats per minute) & $2.56(5.37)$ & 0.05 & $1.02(4.00)$ & 0.27 \\
Respiratory rate (breaths per minute) & $0.53(4.81)$ & 0.63 & $-0.20(6.50)$ & 0.89 \\
Systolic blood pressure (mmHg) & $1.51(5.71)$ & 0.25 & $0.57(3.45)$ & 0.47 \\
Diastolic blood pressure (mmHg) & $1.29(3.04)$ & 0.07 & $0.71(3.23)$ & 0.34 \\
Oxygen saturation (\%) & $-0.60(2.16)$ & 0.17 & $0.14(1.52)$ & 0.68 \\
\hline
\end{tabular}

*One-sample t-test

before the intervention to during the intervention were statistically significant $(p=0.05)$, they were not clinically significant with a mean increase of 2.56 beats per minute. There were no other significant changes in vital signs or oxygen saturations (Table 3).

\section{Feasibility}

There was a total of 268 eligible days for passive range of motion exercise across the cohort. High completion of the exercise protocol $(>75 \%$ of the exercise) across all patients was achieved on $88 \%$ of the eligible days (237) and medium completion (>50\% of the exercise) was achieved on an additional $2 \%$ of the days (5) (Fig 2). The inability of the physical therapist to complete $100 \%$ of all daily repetitions on both upper and lower extremities was largely related to the placement of a peripheral intravenous or arterial line in one of the extremities. On 18/ 268 days, the intervention was deferred by medical or nursing staff, parents, or due to lack of physical therapy staff availability. Birth length, birth weight, gestational age, ICU days, length of stay, and ventilation days were not significantly associated with high versus low completion.

The passive range of motion intervention was stopped a total of 15 times, out of 268 attempts, in 8 of the 20 patients. In one patient who completed 21 days of the intervention, the daily exercise was stopped a total of seven times because of irritability and drop in oxygen saturations. A second patient's

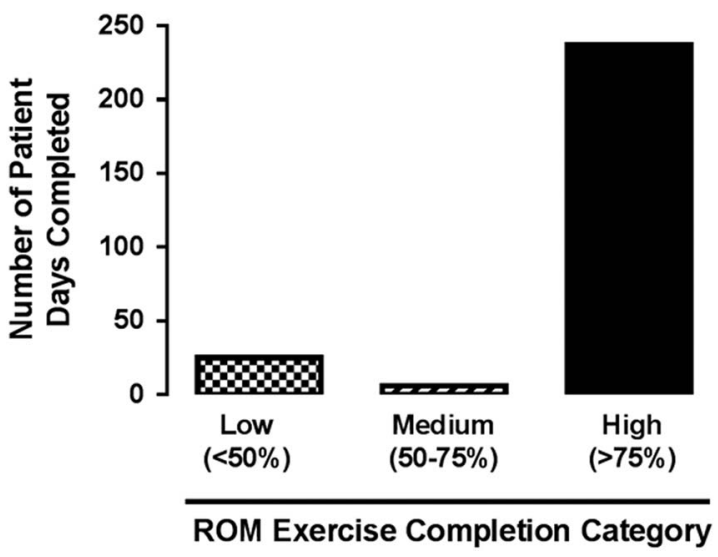

Figure 2.

Feasibility. Determined by the proportion of days where $<50 \%$ (low), $50-75 \%$ (medium) or $>75 \%$ (bigh) of the passive range of motion (ROM) exercises were performed.

parent deferred initiation of the exercise on two occasions as the baby was observed to be distressed when feeding earlier in the day. A third patient became irritable during the intervention, having tolerated it 2 days earlier, and the parent withdrew the patient from the study. In four other patients, the daily exercise was stopped once each because of irritability and heart rate changes $(n=2)$ or infant "state" $(n=2)$. Only one patient was noted to have a chest tube leak, unrelated to the intervention, and thus the exercise was stopped at once. Of the 20 patients, 12 completed all the daily passive range of motion protocol, without any interruptions. 


\section{Anthropometry and growth}

At the pre-Stage II visit, weight-for-age z-scores $(-0.84 \pm 1.2)$ and length-for-age $z$-scores $(-0.83 \pm 1.31)$ were higher than previous reports in Pediatric Heart Network-studied single-ventricle cohorts enrolled in the Single Ventricle Reconstruction and Infant Single Ventricle trials (Fig 2). Compared with the Single Ventricle Reconstruction population, our cohort had less decrease in weight-for-age z-scores from baseline to discharge $(-0.18( \pm 1.49)$ versus $-1.40( \pm 0.82))$ and from baseline to pre-Stage II $(-0.56( \pm 0.81)$ versus $-1.50( \pm 1.14))$. We also noted an improved change in length-for-age z-scores from baseline to discharge $(0.05( \pm 1.22)$ versus -1.3 $( \pm 1.23))$ and baseline to pre-Stage II $(0.05( \pm 1.59)$ versus $-1.48( \pm 1.66)$ ) when compared with the Single Ventricle Reconstruction population. There were no differences between our cohort and the Single Ventricle Reconstruction cohort in changes from discharge to pre-Stage II and no differences in z-score change at any time points between this cohort and the Infant Single Ventricle cohort.

\section{Discussion}

This study is the first to describe the safety and feasibility of a daily passive range of motion exercise protocol in neonates immediately following the Norwood operation for single-ventricle palliation for $\mathrm{CHD}$. The results support that this intervention can be executed safely, without significant adverse events or changes in clinical status, in this high-risk, fragile population. Achievement of the daily passive range of motion exercises was feasible in this population with high completion rates. The pre-Stage II anthropometry in this pilot cohort suggests that passive range of motion should be investigated for its potential to improve growth in single-ventricle $\mathrm{CHD}$.

Although daily compliance with the protocol was high, the duration of the intervention was shorter than anticipated. Not achieving the target of 21 days of passive range of motion per patient was driven by the shorter-than-expected hospital length of stay (median 15 days), rather than intolerance to exercise, as patients were discharged earlier than the average length of stay (24 days) seen in previous Pediatric Heart Network studies involving single-ventricle patients. Moreover, one might speculate that an era effect may impact the length of stay, with the emphasis on high-risk clinics and home monitoring contributing to these babies being discharged earlier. To reach the goal of 21 days, it may be possible to train caregivers to continue the exercise at home. In an exercise-assisted programme for preterm infants, caregivers, mostly mothers, were taught by nurses, occupational therapists, and other healthcare providers to perform daily exercise for 3 weeks with their infants at home. The caregivers were willing and able to perform the exercises correctly and expressed belief that the intervention had positive effects on their babies and on caregiver-infant interactions. ${ }^{24}$ Although interventions in preterm infants have shown promising effects, the benefit may be enhanced when continued at home after discharge.

Movement in utero and early neonatal life may be an important modifier of growth and neurodevelopment. The last trimester of pregnancy sees a striking increase in fetal body mass and physical activity, where the fetus moves in an increasingly restricted intrauterine environment; however, it has been a tenet of neonatal care of high-risk infants that all nongrowth related energy expenditure such as normal activity should be minimised, so that caloric intake would be used primarily for growth ${ }^{27}$ and to avoid the hypoxic episodes that can occur with frequent handling. ${ }^{28}$ The benefit of handling these infants on caregiver-infant interactions likely starts much earlier than at the time of hospital discharge. Infants born with CHD can experience prolonged hospitalisation for weeks or even months, characterised by altered sensory and physical stimulation. Sedation and paralysis may be implemented for respiratory support, haemodynamic instability, or bleeding, further diminishing normal movement. Therefore, it is important to identify strategies to safely increase activity and movement to potentially enhance growth in infants with single-ventricle physiology and prevent neurodevelopmental delays and other adverse outcomes.

Previous studies in preterm and low birth weight infants, excluding CHD infants, have demonstrated that moderate-pressure massages or passive range of motion exercises increase somatic growth when performed in a systematic, standardised manner by trained individuals. ${ }^{17}$ Although the mechanism by which this intervention works on growth is unknown, several hypotheses have been suggested. Some investigators suggest that changes in vagal tone and secretion of catecholamines may play a role ${ }^{29}$ or that increased gastric motility and vagal activity may innervate the digestive system. ${ }^{30}$ Alternatively, weight gain with infant massage may occur as a result of reduced energy expenditure, ${ }^{8}$ despite the observation that infants appeared to be more active during and after the exercise intervention. Field et $\mathrm{al}^{31}$ proposed that massage therapy may lead to increased weight gain by increasing insulin and Insulin-like growth factor-1 levels, as insulin promotes the conversion of glucose to both short-term - glucagon and long-term - lipids/fats - storage. Insulin-like growth factor-1 also plays an important role in 


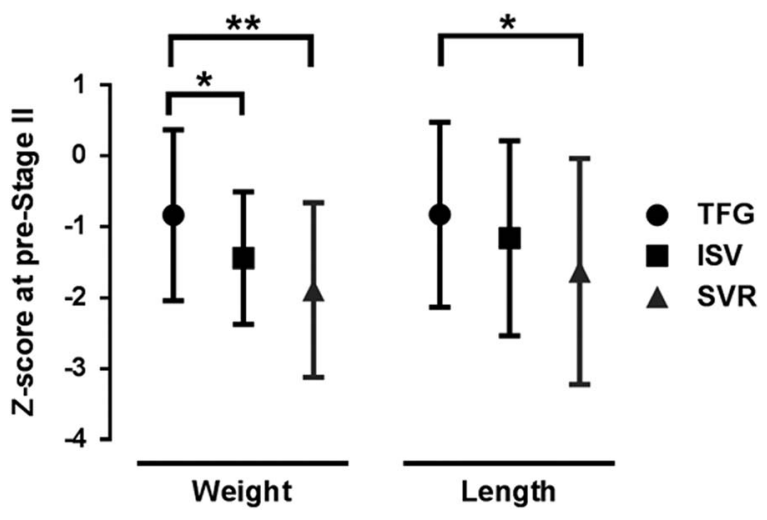

Figure 3.

Preliminary efficacy. Mean weight-for-age z-score and length-forage z-score at 3 months or pre-stage II palliation follow-up for the study cohort (Team for Growth (TFG); $n=18$ ), Infants with Single Ventricle (ISV) $n=56$ and Single Ventricle Reconstruction $n=221(* p<0.05, * * p<0.01)$.

promoting growth by stimulating cell growth and inhibiting apoptosis.

Stress can be common in infants in ICU. This correlates with poor glucose metabolism, leading to hyperglycaemia and insulin resistance, which may be decreased with massage therapy. Massage therapy may increase more restful and calmer states in neonates, likely the result of a parasympathetic response elicited by the stimulation of pressure receptors, which both decreases cortisol levels and increases vagal activity. Guzzetta et $\mathrm{al}^{32}$ found that massaged infants demonstrated significantly decreased cortisol levels at the end of the massage protocol compared with control infants. Increased vagal activity may lead to greater weight gain by increasing gastric activity and promoting the release of insulin.

This study did not include a control group and was not powered for growth outcomes. Nevertheless, we did compare growth in this small cohort with two historical cohorts studied by the Pediatric Heart Network. The two previous trials, the Infant Single Ventricle Trial ${ }^{25}$ and the Single Ventricle Reconstruction Trial, ${ }^{26}$ both enrolled neonates with singleventricle physiology with similar inclusion/exclusion criteria and obtained length/weight at similar time points. Acknowledging that even the small differences in time of enrolment, discharge, and pre-Stage II evaluation may skew growth comparisons, Figure 3 is encouraging in that the neonates in this study were heavier and longer than infants in the other two studies at the pre-Stage II evaluation, even with a median of 15 days of passive range of motion, which was below the target duration. The optimal duration of passive range of motion is unknown. It may be that a longer duration of the exercise protocol is needed to improve or sustain growth as evidenced by no difference between the cohorts in the discharge-topre-Stage II time frame. It may also be that the ICU is the critical window for benefit from passive range of motion exercise. Although the preliminary anthropometric findings are encouraging, appropriately controlled studies are required to understand the efficacy as well as the effect of passive range of motion and handling on cortisol, Insulin-like growth factor-1, and insulin.

\section{Limitations}

This non-randomised study was a pilot to assess the safety and feasibility of a passive range of motion protocol in 20 infants with single-ventricle physiology, and was not powered to evaluate increased growth. It is possible that healthier babies were preferentially invited to participate in this study or parents of healthier babies were more likely to consent, but efforts were made to enroll all babies who met inclusion criteria and none of exclusion criteria.

\section{Conclusions}

A passive range of motion exercise programme is safe and feasible in infants with single-ventricle physiology. Although the mechanisms of action are not well defined, previous studies demonstrate that assisted exercise is a promising strategy for enhancing somatic growth in neonates at risk for growth failure. This study confirms that a once-daily, passive range of motion exercise programme in single-ventricle infants in the immediate postoperative period is safe and can be executed in the demanding environment of the cardiac ICU. Larger studies are required to explore the optimal duration of passive range of motion exercise and its effect on somatic growth and neurobehaviour.

\section{Acknowledgement}

None.

\section{Financial Support}

The project was supported by U01 grants from the National Heart, Lung, and Blood Institute (HL068269, HL068270, HL068279, HL068281, HL068285, HL068292, HL068290, HL068288, HL085057). Its contents are solely the responsibility of the authors and do not necessarily represent the official views of the National Heart, Lung, and Blood Institute. Clinical Trial Registration no: NCT01917084, URL: https://clinicaltrials.gov/ct2/ show/NCT01917084. 


\section{Conflicts of Interest}

The authors have no conflicts of interest.

\section{References}

1. Owens JL, Musa N. Nutrition support after neonatal cardiac surgery. Nutr Clin Pract 2009; 24: 242-249.

2. Menon G, Poskitt EM. Why does congenital heart disease cause failure to thrive? Arch Dis Child 1985; 60: 1134-1139.

3. Burch PT, Gerstenberger E, Ravishankar C, et al. Longitudinal assessment of growth in hypoplastic left heart syndrome: results from the single ventricle reconstruction trial. J Am Heart Assoc 2014; 3: e000079.

4. Williams RV, Zak V, Ravishankar C, et al. Factors affecting growth in infants with single ventricle physiology: a report from the Pediatric Heart Network Infant Single Ventricle Trial. J Pediatr 2011; 159: 1017-1022.e2.

5. Vogt KN, Manlhiot C, Van Arsdell G, Russell JL, Mital S, McCrindle BW. Somatic growth in children with single ventricle physiology impact of physiologic state. J Am Coll Cardiol 2007; 50: 1876-1883.

6. Pemberton VL, McCrindle BW, Barkin S, et al. Report of the National Heart, Lung, and Blood Institute's Working Group on obesity and other cardiovascular risk factors in congenital heart disease. Circulation 2010; 121: 1153-1159.

7. Leitch CA, Karn CA, Peppard RJ, et al. Increased energy expenditure in infants with cyanotic congenital heart disease. J Pediatr 1998; 133: 755-760.

8. Lahat S, Mimouni FB, Ashbel G, Dollberg S. Energy expenditure in growing preterm infants receiving massage therapy. J Am Coll Nutr 2007; 26: 356-359.

9. Lambert LM, Pike NA, Medoff-Cooper B, et al. Variation in feeding practices following the Norwood procedure. J Pediatr 2014; 164: 237-242.e1.

10. Anderson JB, Beekman RH 3rd, Border WL, et al. Lower weightfor-age z score adversely affects hospital length of stay after the bidirectional Glenn procedure in 100 infants with a single ventricle. J Thorac Cardiovasc Surg 2009; 138: 397-404.e1.

11. Cohen MS, Zak V, Atz AM, et al. Anthropometric measures after Fontan procedure: implications for suboptimal functional outcome. Am Heart J 2010; 160: 1092-8-1098.e1.

12. Miller TA, Zak V, Shrader P, et al. Growth asymmetry, head circumference, and neurodevelopmental outcomes in infants with single ventricles. J Pediatr 2016; 168: 220-225.e1.

13. Franz AR, Pohlandt F, Bode H, et al. Intrauterine, early neonatal, and postdischarge growth and neurodevelopmental outcome at 5.4 years in extremely preterm infants after intensive neonatal nutritional support. Pediatrics 2009; 123: e101-e109.

14. Chan GM, Armstrong C, Moyer-Mileur L, Hoff C. Growth and bone mineralization in children born prematurely. J Perinatol 2008; 28: 619-623.

15. Moyer-Mileur L, Luetkemeier M, Boomer L, Chan GM. Effect of physical activity on bone mineralization in premature infants. J Pediatr 1995; 127: 620-625.
16. Moyer-Mileur LJ, Brunstetter V, McNaught TP, Gill G, Chan GM. Daily physical activity program increases bone mineralization and growth in preterm very low birth weight infants. Pediatrics 2000; 106: 1088-1092.

17. Field T, Diego M, Hernandez-Reif M. Preterm infant massage therapy research: a review. Infant Behav Dev 2010; 33: $115-124$.

18. Rutherford M, Ramenghi LA, Edwards AD, et al. Assessment of brain tissue injury after moderate hypothermia in neonates with hypoxic-ischaemic encephalopathy: a nested substudy of a randomised controlled trial. Lancet Neurol 2010; 9: 39-45.

19. Dieter JN, Field T, Hernandez-Reif M, Emory EK, Redzepi M. Stable preterm infants gain more weight and sleep less after five days of massage therapy. J Pediatr Psychol 2003; 28: 403-411.

20. Ferber SG, Kuint J, Weller A, et al. Massage therapy by mothers and trained professionals enhances weight gain in preterm infants. Early Hum Dev 2002; 67: 37-45.

21. Kuhn CM, Schanberg SM, Field T, et al. Tactile-kinesthetic stimulation effects on sympathetic and adrenocortical function in preterm infants. J Pediatr 1991; 119: 434-440.

22. Als H, Gilkerson L, Duffy FH, et al. A three-center, randomized, controlled trial of individualized developmental care for very low birth weight preterm infants: medical, neurodevelopmental, parenting, and caregiving effects. J Dev Behav Pediatr 2003; 24: 399-408.

23. Control CfD. Retrieved 10 February 2017 from http://www.cdc.gov/ mmwr/preview/mmwrhtml/rr5909a1.htm. September 10, 2010.

24. McQueen D, Lakes K, Rich J, et al. Feasibility of a caregiverassisted exercise program for preterm infants. J Perinat Neonatal Nurs 2013; 27: 184-192.

25. Hsu DT, Mital S, Ravishankar C, et al. Rationale and design of a trial of angiotensin-converting enzyme inhibition in infants with single ventricle. Am Heart J 2009; 157: 37-45.

26. Ohye RG, Sleeper LA, Mahony L, et al. Comparison of shunt types in the Norwood procedure for single-ventricle lesions. $\mathrm{N}$ Engl J Med 2010; 362: 1980-1992.

27. Langer VS. Minimal handling protocol for the intensive care nursery. Neonatal Netw 1990; 9: 23-27.

28. Long JG, Philip AG, Lucey JF. Excessive handling as a cause of hypoxemia. Pediatrics 1980; 65: 203-207.

29. Acolet D, Modi N, Giannakoulopoulos X, et al. Changes in plasma cortisol and catecholamine concentrations in response to massage in preterm infants. Arch Dis Child 1993; 68: 29-31.

30. Diego MA, Field T, Hernandez-Reif M, Deeds O, Ascencio A, Begert G. Preterm infant massage elicits consistent increases in vagal activity and gastric motility that are associated with greater weight gain. Acta Paediatr 2007; 96: 1588-1591.

31. Field T, Diego M, Hernandez-Reif M, et al. Insulin and insulin-like growth factor-1 increased in preterm neonates following massage therapy. J Dev Behav Pediatr 2008; 29: 463-466.

32. Guzzetta A, Baldini S, Bancale A, et al. Massage accelerates brain development and the maturation of visual function. J Neurosci 2009; 29: 6042-6051. 